\title{
Electrospray Mass Spectrometry of NeuAc Oligomers Associated with the C Fragment of the Tetanus Toxin
}

\author{
Maria C. Prieto Conway \\ Chemistry and Materials Science Directorate, Lawrence Livermore National Laboratory, Livermore, \\ California, USA
}

\author{
Randy M. Whittal, Michael A. Baldwin, and A. L. Burlingame \\ Mass Spectrometry Facility, Department of Pharmaceutical Chemistry, University of California, \\ San Francisco, California, USA
}

\section{Rod Balhorn}

Biology and Biotechnology Research Program, Lawrence Livermore National Laboratory, Livermore, California, USA

\begin{abstract}
The Clostridial neurotoxins, botulinum and tetanus, gain entry into motor neurons by binding to the sialic or $\mathrm{N}$-acetylneuraminic acid (NeuAc) residues of gangliosides and specific protein receptors attached to the cell's surface. While the C-fragment of tetanus toxin (TetC) has been identified to be the ganglioside binding domain, remarkably little is known about how this domain discriminates between the structural features of different gangliosides. We have used electrospray ionization mass spectrometry (ESI-MS) to examine the formation of complexes between TetC and carbohydrates containing NeuAc groups to determine how NeuAc residues contribute to ganglioside binding. ESI-MS was used to obtain an estimate of the dissociation constants ( $\mathrm{K}_{\mathrm{D}}$ values) for TetC binding to a number of related NeuAc-containing carbohydrates (sialyllactose and disialyllactose), as well as six (NeuAc) oligomers $(n=1-6)$. $\mathrm{K}_{\mathrm{D}}$ values were found to range between $\sim 10-35 \mu \mathrm{M}$. The strength of the interactions between the $\mathrm{C}$ fragment and (NeuAc) $)_{n}$ are consistent with the topography of the targeting domain of tetanus toxin and the nature of its carbohydrate binding sites. These results suggest that the targeting domain of tetanus toxin contains two binding sites that can accommodate NeuAc (or a dimer) and that NeuAc may play an important role in ganglioside binding and molecular recognition, a process critical for normal cell function and one frequently exploited by toxins, bacteria, and viruses to facilitate their entrance into cells. (J Am Soc Mass Spectrom 2006, 17, 967-976) (C) 2006 American Society for Mass Spectrometry
\end{abstract}

$\mathrm{B}$ oth botulinum and tetanus neurotoxins interact with motor neurons, blocking acetylcholine release and causing muscle paralysis and death (for an excellent review, see Niemann, 1991, [1]). The first step required for toxin entrance into neurons is the recognition of gangliosides at the cell surface by the receptor-binding domain of the heavy chain $[2,3]$. Following the binding of the toxin to the cell surface, an endosome forms around the toxin, and the translocation domain of the toxin transfers the catalytic domain, a zinc protease, into the cell. Once inside the cell, the protease cleaves one of three specific proteins involved

Published online May 19, 2006

Address reprint requests to Dr. R. Balhorn, Biology and Biotechnology Research Program, Lawrence Livermore National Laboratory, 7000 East Avenue, Livermore, CA 94550, USA. E-mail: balnorn2@llnl.gov

* Present address: Department of Chemistry, University of Alberta, Edmonton, Alberta T6G 2G2, Canada. in neurotransmitter release, depending on the toxin type.

The targeting domain of the tetanus toxin (TeNT) has been identified as the $51 \mathrm{kDa}$ carboxy-terminal peptide of the heavy chain [4], called the C fragment. While there is considerable evidence indicating that gangliosides are one type of cell surface receptor targeted by this domain, multiple receptors may be required for cell recognition and binding. It has been suggested that protein receptors may contribute to more efficient toxin binding [5] and provide neurospecificity. In certain cases, such as the botulinum neurotoxin, different serotypes appear to exhibit a preference for different types of gangliosides or protein receptors. The strongest and hence most specific ganglioside association with tetanus toxin occurs with $\mathrm{G}_{\mathrm{T1b}}$, a trisialo sphingolipid with a branched carbohydrate structure containing a single NeuAc on one arm and a NeuAc dimer on the other (Table 1). 
Table 1. Ganglioside structures. Carbohydrate sections tested for binding to TetC appear in bold



Electrospray ionization (ES) has been established as a soft-ionization technique for introducing noncovalent complexes into the gas phase [6-11], and previous studies have demonstrated the utility of ESI MS for determining affinity constants $[12,13]$ of protein-ligand complexes. These experiments have evoked considerable discussion in the literature about whether or not the gas phase is a valid representation of solution conditions that govern the rates of association (or dissociation) between proteins and ligands. The conclusions that have been drawn from these discussions are that the bound state is thermodynamically favored in the gas phase for noncovalent complexes dominated by ionic or polar interactions. Conversely, nonpolar or hydrophobic interactions between molecules are favored as dissociated entities in the gas phase [13-15]. For this reason, ESI MS is not recommended as the method of choice studying protein/ligand interactions $[12,15]$. The specific interactions taking place in this study occur between the negatively charged carboxylic groups of the sialic acids and positive (or partially positive) regions on the toxin's surface. Based on the conclusions that have been drawn from previous work, it appears reasonable to assume such a complex could maintain its conformation throughout the ionization process and this MS study might be expected to provide 
dissociation constants similar to those measured in solution. The use of ESI MS for measuring affinity constants in complexes stabilized by hydrophobic interactions is possible, but a different approach may be required to analyze the data $[12,13]$.

It is also important when using ESI-MS for dissociation constant measurements to make certain that the signal intensities reflect the concentrations of the components and complexes in solution. By studying the response of proteins that do not interact to form complexes, ion intensities in the gas phase have been found to be approximately proportional to the protein's concentration in solution [16]. The proportionality between solution concentrations and ion abundances in the gas phase is determined by transfer coefficients for both protein and complex during ionization. Transfer coefficients of protein ligand complexes formed with small ligands would be expected to have values similar to those of the unbound protein, and the two would cancel out when the intensity ratios (complex/protein) are considered [16]. The glycolipids that bind to the tetanus $\mathrm{C}$ fragment have masses in the $1 \mathrm{k}$ to $3 \mathrm{kDa}$ range. The ligands used in this study were slightly smaller, in the range of $300 \mathrm{Da}$ to $2 \mathrm{kDa}$, and small compared to the 51 $\mathrm{kDa} C$ fragment. Therefore, TetC-carbohydrate/ganglioside complexes should be "protein-like", with ionization efficiencies similar to the protein, even though carbohydrates and proteins ionize by different mechanisms. These two factors, the formation of complexes with strong to medium binding affinities and similar ionization efficiencies, favor the use of intensity ratios for the measurements of dissociation constants by ESIMS.

Nondenatured proteins in electrospray ionize by a process that is referred to as a charged residue model (CRM) during droplet evaporation, as opposed to small inorganic ions, which ionize by the ion evaporation model (IEM) [17]. Concerns have been raised about the possibility that complex formation may be artificially induced during the ES process [16]. One concern in the CRM is that the increase in reactant concentrations during droplet evaporation might shift the equilibrium toward complex formation. This concern may be valid when larger droplets are used due to longer evaporation times. However, droplets with diameters of 1 to 10 $\mu \mathrm{m}$ have been shown to evaporate in less than $1 \mathrm{~ms}$, faster than the rate of equilibrium dissociation [18]. Another concern is that ligands may bind randomly and nonspecifically to the protein. These interactions would likely be weaker and nonspecific therefore, they would not be expected to survive the declustering clean-up step before mass analysis.

Previously, we used ESI-MS to screen a group of ligands to identify those that bound to TetC. The molecules tested were identified as potential binders by screening a ligand database and assessing the ability of each ligand to form a complex with TetC using computational docking tools [19]. In the present work, we show that ESI-MS can be used to measure equilibrium dissociation constants $\left(K_{D}\right)$ between TetC and NeuAccontaining carbohydrates. Dissociation constants were measured for a series of NeuAc oligomers, (NeuAc) with $n=1$ to 6 , to assess the validity of the technique for discerning small changes in binding strengths. Two NeuAc-containing carbohydrates and one non-binding control, which are subsets of the ganglioside structures that bind with different affinities to TetC, were also tested. The protein concentration was kept constant in the experiments with the ligand titrated in at various concentrations. The ratio of the signal intensities of the peak heights for the protein alone and the proteincarbohydrate complex as determined by ESI MS (twosignal approach) was used for determining dissociation constants. These ESI-MS results are discussed in light of published results obtained by other solution-based techniques to identify ganglioside structural features that may contribute to their binding to tetanus neurotoxin.

\section{Experimental}

\section{Materials}

A recombinant form of TetC was purchased from Roche Diagnostics Corp. (Indianapolis, IN). The NeuAc monomer (309.3 Da), and the oligomeric dimer (644.5 Da), trimer (957.7 Da), tetramer (1271 Da), pentamer (1584.2 $\mathrm{Da})$, and hexamer (1897.5 Da), were obtained from Calbiochem-Novabiochem Corp. (La Jolla, CA) as the sodium salts. $\beta$ D-Gal(1-3)D-GalNAc (383.4 Da) and the sodium salts of oligosaccharides occurring in the gangliosides $\mathrm{GM}_{3}$ and $\mathrm{GT}_{1 \mathrm{~b}}, 3^{\prime}$-N-acetylneuraminyl lactose (3'-sialyllactose, 655.5 Da), di-N-acetylneuraminyl lactose (disialyllactose, $963.7 \mathrm{Da}$ ) were purchased from Sigma Chemical Co. (St. Louis, MO).

\section{Instrumentation}

Samples were analyzed by ESI-MS on a Mariner orthogonal acceleration time-of-flight (oa-TOF) instrument (Applied Biosystems, Framingham MA). The use of this instrument to monitor noncovalent interactions has been described previously [20, 21]. The singly and doubly charged ions of Gramicidin S were used to calibrate the mass scale, typically at the start of each day. Freshly mixed protein/carbohydrate samples were introduced by infusion from a syringe pump through fused silica capillaries (60 and $25 \mu \mathrm{m}$ i.d.) at $1 \mu \mathrm{L} / \mathrm{min}$. Once a stable signal was obtained, spectra were accumulated over a period of $2 \mathrm{~min}$. All spectra were acquired at room temperature to minimize dissociation of the complexes. Instrument settings, such as gas flow rates, number of scans, spray tip potential, and declustering or nozzle potentials were optimized and kept constant for each set of experiments corresponding to a specific complex. The nozzle or declustering potential was adjusted in the range 50 to $300 \mathrm{~V}$ to obtain a qualitative estimate of the strength and nature of the 


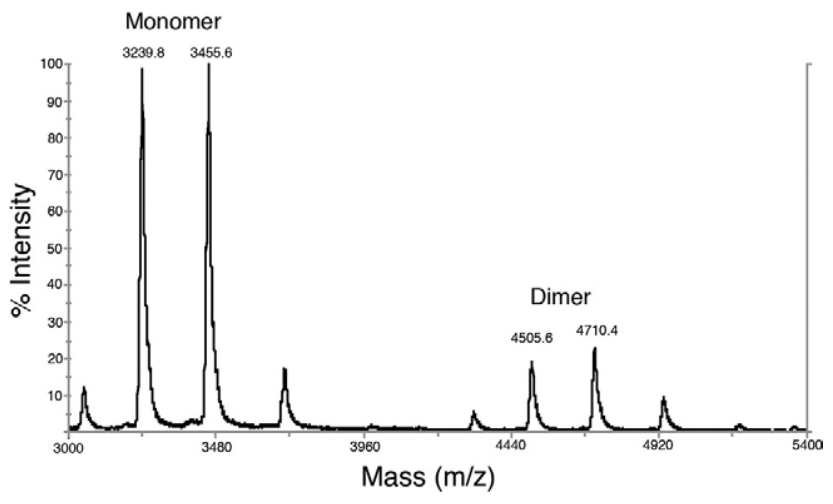

Figure 1. ESI MS spectrum from $7 \mu \mathrm{M}$ solution of TetC, recorded with a declustering voltage of $300 \mathrm{~V}$, showing the monomer (M) multiply charged envelope with $z$ equal to $17,16,15$, and 14 , and the dimer (2M) at higher $\mathrm{m} / \mathrm{z}$ with $\mathrm{z}$ equal to $24,23,22$, and 21 .

noncovalent interactions. Multiply charged spectra were deconvoluted using the Biospec Data Explorer software supplied with the mass spectrometer.

\section{Complex Formation}

The TetC protein (recombinant and purified by acid precipitation) was dissolved in filtered deionized water (18 Mohm $\mathrm{cm}^{-1}$, Millipore, Billerica, MA) and centrifuged to remove the insoluble residue that forms at $\mathrm{pH}$ $<9$. Aliquots of the supernatant were hydrolyzed in $\mathrm{HCl}$ and the protein concentration was determined by quantitative amino acid analysis (Protein Structure Laboratory, University of California, Davis, CA). UV-VIS absorption measurements were used to accurately determine the protein concentration in each experiment.

Each experiment was conducted using a fixed concentration of protein, and the concentration of the ligand was varied. Twenty $\mu \mathrm{l}$ samples were prepared for analysis. The TetC sample was diluted to a final concentration of 5 to $10 \mu \mathrm{M}$ in $3 \mathrm{mM}$ aqueous ammonium acetate $(\mathrm{pH} \sim 7.6)$ and $12 \%$ methanol. Stock carbohydrate samples were dissolved in water and final dilutions were made in $3 \mathrm{mM}$ aqueous ammonium acetate $(\mathrm{pH} \sim 7.6)$ and $12 \%$ methanol. The $\mathrm{Na}^{+}$salts were not removed from the ligands. The ratios of protein:ligand were varied over a range of 1:1 to 1:10.

\section{Data Analysis}

$$
\begin{gathered}
\text { Protein }+\underset{\mathrm{k}_{d}}{\stackrel{\mathrm{k}_{a}}{\rightleftharpoons} \text { Liganplex }} \\
\mathrm{K}_{\mathrm{D}}=\frac{\mathrm{k}_{\mathrm{d}}}{\mathrm{k}_{\mathrm{a}}}=\frac{[\text { Protein }][\text { Ligand }]}{[\text { Complex }]}
\end{gathered}
$$

The equations describing simple binding kinetics are given by eqs 1 and 2, where $k_{a}$ and $k_{d}$ are rate constants for the association and dissociation, respectively, of the complex, $\mathrm{K}_{\mathrm{D}}$ is the equilibrium dissociation constant for dissociation, and [Protein], [Ligand], and [Complex] are the concentrations of the corresponding species at equilibrium. A large $K_{D}$ indicates that $k_{d}$ is greater than $k_{a}$, which means the ligand has a weaker affinity for the protein and the complex dissociates more readily. A small $K_{D}$ indicates a stronger affinity between protein and ligand.

If we assume a single complex is formed and the ion peak intensities in an ESI mass spectrum are proportional to solution concentrations of the components at equilibrium, the ratio [Protein]/[Complex] can be derived from the ratio of the peak heights for the protein alone and the protein-carbohydrate complex. The dissociation constant can then be obtained from a plot of this ratio versus $1 /$ [ligand] [7]. In such experiments, the transfer coefficient, a proportionality constant that is affected by analyte mass and instrumental conditions, cancels out of the equation when the masses of the protein and the complex are similar $[6,16]$.

\section{Results and Discussion}

\section{ESI-MS of TetC}

A typical ESI-MS spectrum of TetC shows two species present, a monomer and a dimer (Figure 1). The mass deconvolution spectra for these two peaks (Figure 2) yield masses of 51,819 and 103,606 for the protein monomer and dimer components, respectively. The molecular weight of this recombinant protein based on its amino acid sequence is 51,758 Da. The observed differences in mass between the molecular weights derived from the TetC sequence and our measured



Figure 2. Deconvolution of separate regions of the ESI MS spectra of TetC, corresponding to (a) M, and (b) $2 \mathrm{M}$. 
values (61 Da for the monomer, $90 \mathrm{Da}$ for the dimer) are small and appear to be attributed to tightly bound counterions (acetate, ammonium, sodium) or water molecules.

\section{TetC Dimerization}

Following its binding to gangliosides and a protein receptor, TeNT oligomerizes and is pulled into an endosome where it forms a channel or pore in the cell membrane through which it transfers its catalytic domain [22-25]. Native gel electrophoresis and chemical crosslinking experiments have shown that TeNT forms dimers and trimers in solution [26]. Previous cryoelectron microscopy [23] and ion conductance [24] studies have also shown that both botulinum toxin and TeNT can form tetrameric channels in neuronal membranes. However, higher order TetC oligomers, such as trimers or tetramers, were not observed in the ESI-MS data presented here, probably because of the mass range limitations of the Mariner mass spectrometer used.

The TetC dimer association that was observed was further characterized by ESI-MS. The first step was to determine if the dimer reflected a valid protein state as would be observed in solution or if it was simply an artifact of the electrospray process. Data obtained on TetC samples using a declustering potential of $300 \mathrm{~V}$ provided the best signal to noise $(\mathrm{S} / \mathrm{N})$ and highest resolution spectra $(\mathrm{m} / \mathrm{dm}=216$ at $300 \mathrm{~V})$. A monomer was the predominantly form observed under these experimental conditions, even at higher TetC concentrations (Figure 3). An increase in dimer formation was only observed at lower declustering voltages suggesting that hydrophobic interactions are the most likely interactions that stabilize the formation of the dimer. In this case, addition of a hydrophobic solvent, such as methanol would be expected to break apart the dimer. Increasing the methanol concentration did not affect the protein (monomer) envelope distribution. However the protein dimer peak disappeared from the mass spectrum when the methanol concentration reached 40\% (Figure 4). These results show that the dimer is not an artifact produced by the electrospray process and that its stability is disrupted by moderate methanol concentrations. The observation that the dimer is always observed in lower abundance than the monomer in the ESI-MS spectra suggests that the stability of the hydrophobic interactions is more easily disrupted in the gas phase [13-15].

\section{Characterization of the TetC/Ligand Complexes}

One goal of this work was to identify the number of sialic groups that bind to TetC and obtain an estimate for the distance between their binding sites. This followed from previously published work in which the number and branching (spacing) of sialic groups in gangliosides were used to explain variability in mea-

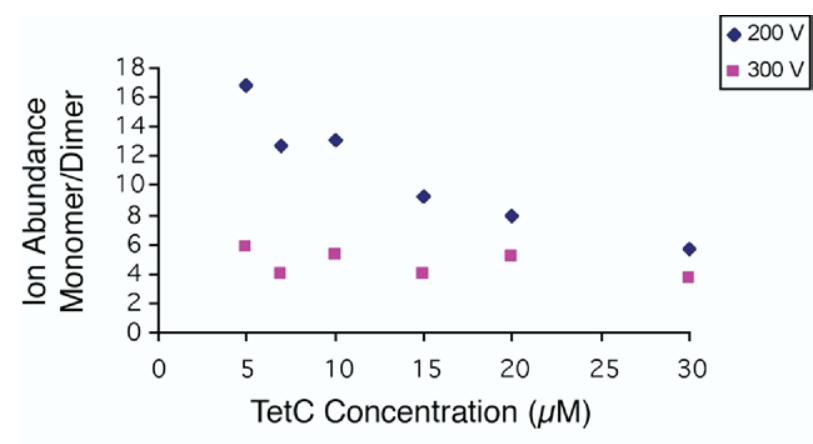

\begin{tabular}{|c|c|c|c|}
\hline $\begin{array}{c}\text { Tetanus C } \\
\text { fragment } \\
\mu \mathrm{M}\end{array}$ & $\begin{array}{c}\text { Declustering } \\
\text { Potential }\end{array}$ & $\begin{array}{c}\text { Monomer } \\
\text { Ion } \\
\text { Abundance }\end{array}$ & $\begin{array}{c}\text { Dimer Ion } \\
\text { Abundance }\end{array}$ \\
\hline 5 & 200 & 1375.7 & 82 \\
7 & 200 & 2364.5 & 186 \\
10 & 200 & 2220.5 & 168.8 \\
15 & 200 & 1631.5 & 176.2 \\
20 & 200 & 793.1 & 99.9 \\
30 & 200 & 637.1 & 110.7 \\
\hline 5 & 300 & 2492.2 & 430 \\
7 & 300 & 3282.3 & 835.6 \\
10 & 300 & 1805.7 & 341.7 \\
15 & 300 & 2414.1 & 600.6 \\
20 & 300 & 850.4 & 164.1 \\
30 & 300 & 1529.8 & 411.4 \\
\hline
\end{tabular}

Figure 3. TetC concentration versus ion abundance of monomer/dimer at declustering voltages of 200 and $300 \mathrm{~V}$.

sured affinity constants. Since the first step in the recognition process is that between one $\mathrm{C}$ fragment and its cell receptor, we assumed that the TetC monomer represented a good model for the simplest interaction that would still be physiologically significant.

Carbohydrates in gangliosides that preferentially bind to the Clostridial neurotoxins typically contain a single (NeuAc) $)_{2}$ separated from another NeuAc (GT1b, $\mathrm{GD} 1 b)$ or $(\mathrm{NeuAc})_{2}\left(\mathrm{G}_{\mathrm{O} 1 \mathrm{~b}}\right)$ residue by other sugar residues (Table 1). The ligands tested for noncovalent complex formation with TetC are listed in Table 2, along with the experimentally determined dissociation constants measured at declustering voltages in the range of 200 to $250 \mathrm{~V}$.

Only ion abundances from the same spectrum were compared in each experiment. The standard deviations were obtained from the regression analysis, and only one binding experiment was repeated ([NeuAc $]_{4}$ ligand) to obtain $K_{D}$ values. In this case, the replicate $K_{D} S$ were 10 and $14 \mu \mathrm{M}$. However, each titration experiment was repeated at three declustering voltages: 200, 250, and 300 Volts. Three linear regressions (one at each voltage) were calculated for each ligand. The measured dissociation constants obtained from each experiment increased linearly with increasing declustering voltage (data not shown). This trend has been previously ob- 


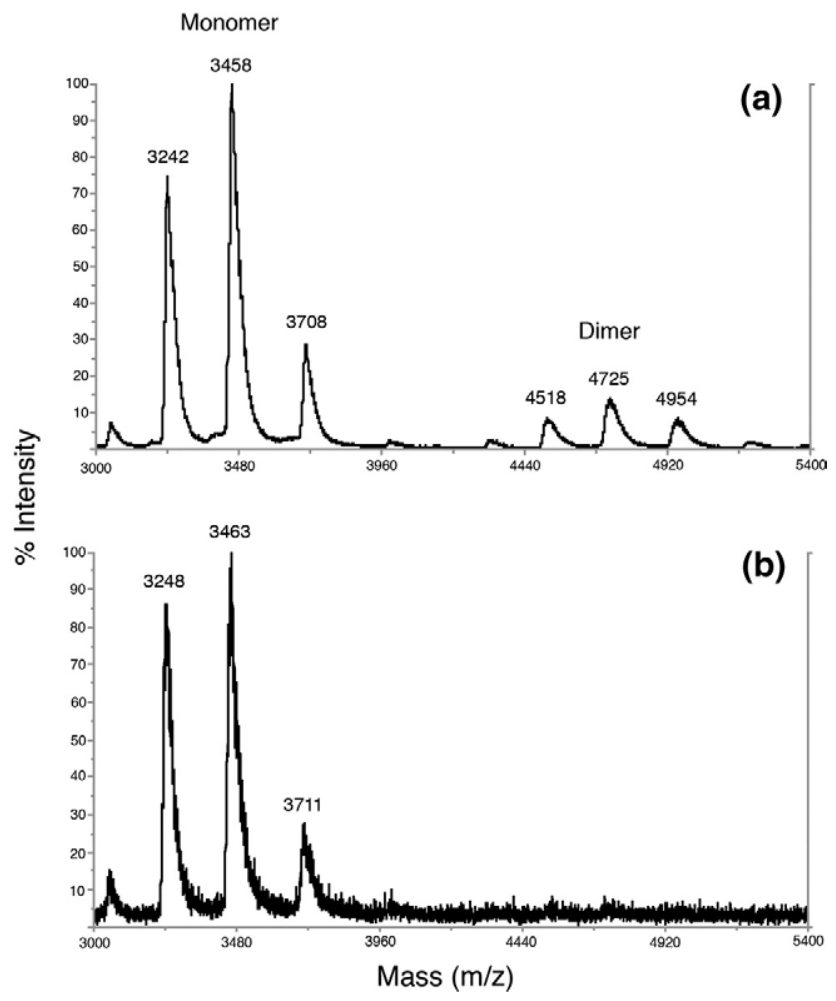

Figure 4. ESI MS spectra of TetC in, (a) 10\% methanol, showing both $\mathrm{M}$ and $2 \mathrm{M}$ peaks, and (b) $40 \%$ methanol, showing the disappearance of the $2 \mathrm{M}$ peak. The protein concentration was 8.6 $\mathrm{mM}$ in $3 \mathrm{mM}$ ammonium acetate, $\mathrm{pH}$ 7.5. The declustering voltage was $200 \mathrm{~V}$ in both cases.

served in a triple quadrupole MS experiment and was attributed to increased collision-induced dissociation of the complex ions at higher tube lens voltages [27]. Repeating the MS measurement at a different declustering voltage for each ligand concentration provided confidence as to the reproducibility of the measurements.

The amount of ligand-bound TetC (normalized to unbound protein) increased linearly, in proportion to ligand concentration, up to a saturation point. Increasing the ligand concentration beyond this point did not produce additional complex and an asymptote was reached (data not shown). In each experiment, the ligand concentration was kept within the linear region of the saturation binding curve [28].

A typical ESI-MS spectrum after mass deconvolution in which both the unbound and ligand-bound TetC peaks can be distinguished is shown in Figure 5. A plot and linear regression of eq 2 for the TetC-(NeuAc) complex is shown in Figure 6, and the dissociation constants obtained from the slope of the line (eq 2) are listed in Table 2.

All the NeuAc-containing ligands bound to TetC in the gas phase without dissociating at high declustering voltages (200-300 V), indicating that the complexes were primarily stabilized by electrostatic interactions [29]. The experimental control, $\beta$ D-Gal(1-3)D-GalNAc, the terminal carbohydrate in the ganglioside $G_{M 1}$ that lacks a NeuAc group, did not form a complex as determined by ESI-MS. However, Gal-GalNAc was shown to be required for binding to TeNT [34].

Complexes (normalized to unbound protein) were observed to be most abundant at a skimmer voltage of $\sim 200 \mathrm{~V}$ (data not shown). Inspection of the low mass region gave an indication of the amount of unbound ligand remaining. The lowest abundance of free ligand in the mass spectrum was observed at skimmer voltages of 150 to $200 \mathrm{~V}$, and the concentration of unbound ligand increased with increasing skimmer voltage, suggesting that the majority of the ligand remained bound to TetC at $\sim 200 \mathrm{~V}$. This is consistent with the idea that an increase in the number of collisions between ions and background neutral gases might favor products other than the TetC-ligand complex [27].

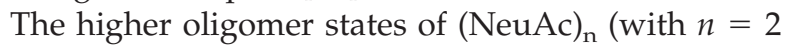
to 5) all formed complexes with the TetC dimer. Fewer data points were generated for the linear regression from these experiments due to the lower ion abundance and poor resolution in that $\mathrm{m} / \mathrm{z}$ region. Ligand binding to the TetC dimer was only observed at the highest concentrations of ligand. The affinity between the TetC dimer and ligands was observed to be lower than with the protein monomer (i.e., weaker binding), as measured by a larger $\mathrm{K}_{\mathrm{D}}$. However, since a sufficient number of reliable measurements could not be obtained, the results are not reported.

Table 2. Ligands tested for noncovalent complex formation with TetC. Spectra were deconvoluted, unless noted otherwise. For each carbohydrate, repeat measurements were made at a constant voltage for different concentrations of ligands, and the regression analysis of the line was used to obtain the standard deviation of the $\mathrm{K}_{\text {dissociation }}$ (see results section for more details)

\begin{tabular}{|c|c|c|}
\hline Carbohydrate & $\begin{array}{l}\text { Declustering } \\
\text { voltage }\end{array}$ & $\begin{array}{l}\mathrm{K}_{\text {dissociation }} \\
\quad(\mu \mathrm{M})\end{array}$ \\
\hline \multirow[t]{2}{*}{${ }^{*} \mathrm{NeuAc}$} & $200 \mathrm{~V}$ & $13 \pm 2^{a}$ \\
\hline & $250 \mathrm{~V}$ & $15 \pm 2$ \\
\hline$(\mathrm{NeuAc})_{2}$ & $200 \mathrm{~V}$ & $21 \pm 4$ \\
\hline$(\mathrm{NeuAc})_{3}$ & $200 \mathrm{~V}$ & $33 \pm 4$ \\
\hline$(\mathrm{NeuAc})_{4}$ & $200 \mathrm{~V}$ & $12 \pm 2^{b}$ \\
\hline$(\mathrm{NeuAc})_{5}$ & $200 V$ & $23 \pm 3$ \\
\hline$(\mathrm{NeuAc})_{6}$ & $200 \mathrm{~V}$ & $11 \pm 4^{a}$ \\
\hline $\begin{array}{l}\text { Sialyllactose }\left(\mathrm{G}_{\mathrm{M} 3} \text { section; }\right. \\
\text { one NeuAc acid) }\end{array}$ & $200 \mathrm{~V}$ & $11 \pm 2$ \\
\hline $\begin{array}{l}\text { Disialyllactose }\left(\mathrm{G}_{\mathrm{T} 1 \mathrm{~b}}\right. \\
\text { section; two NeuAc } \\
\text { acids) }\end{array}$ & $200 \mathrm{~V}$ & $23 \pm 5^{a}$ \\
\hline $\begin{array}{l}\text { Ganglioside control: } \beta \text {-D- } \\
\text { Gal }(\alpha 1-3) \text { GalNAc }\end{array}$ & $\begin{array}{l}\text { 50-250V } \\
\text { (declustering } \\
\text { voltages } \\
\text { tested) }\end{array}$ & $\begin{array}{l}\text { No } \\
\text { binding }\end{array}$ \\
\hline
\end{tabular}

${ }^{*}$ NeuAc $=$ sialic acid.

${ }^{a}$ spectra not deconvoluted. Note that when available, data from both raw data and deconvoluted spectra were in good agreement.

baverage of two separate binding experiments gave similar results. 

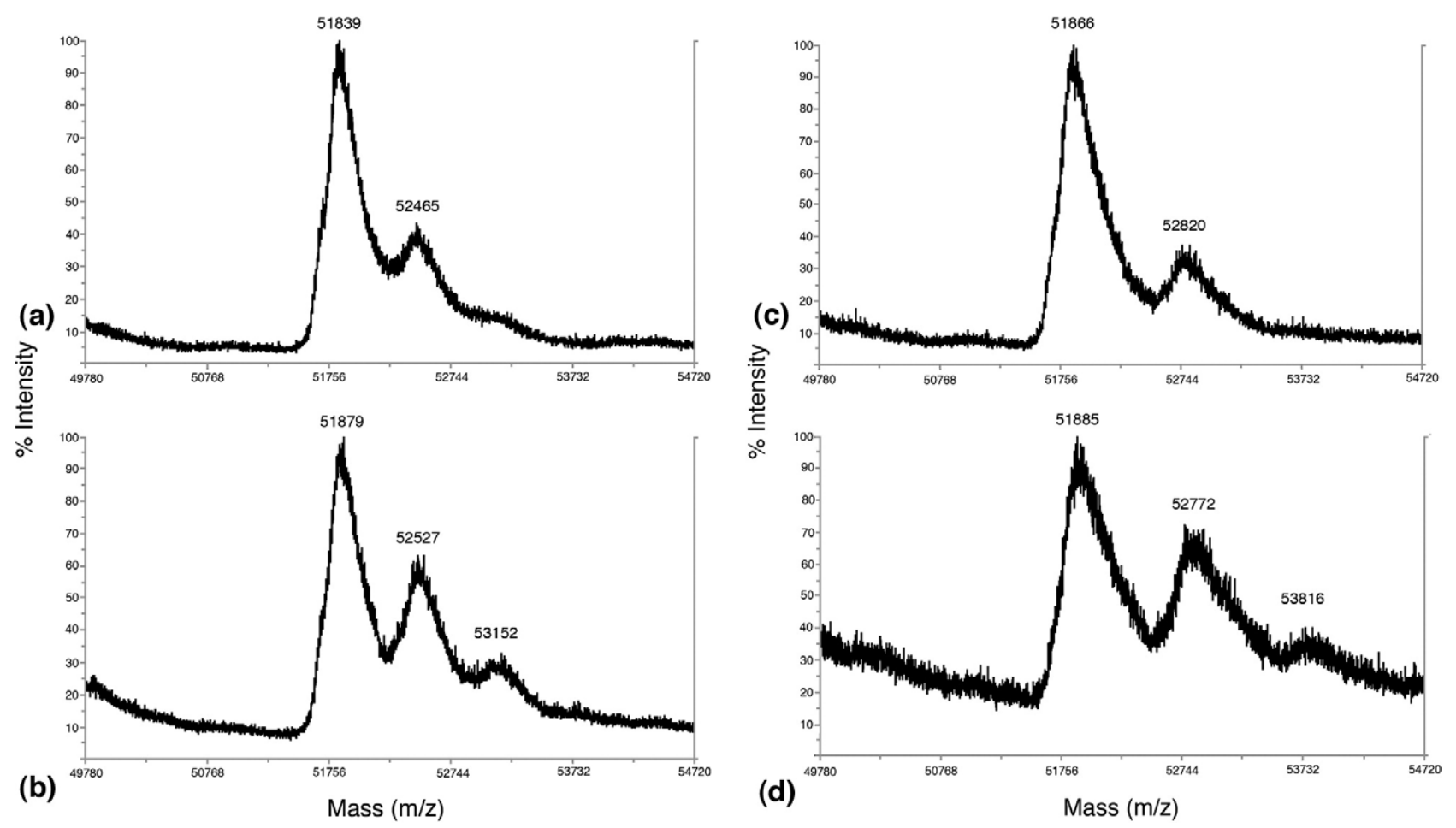

Figure 5. Deconvoluted spectra recorded with a declustering voltage of $200 \mathrm{~V}$, showing complex formation between the TetC and NeuAc dimers and trimers. (a) 1:4 TetC:(NeuAc) ${ }_{2}$; (b) 1:8 TetC: $(\mathrm{NeuAc})_{2} ;$ (c) 1:3 TetC:(NeuAc$)_{3} ;$ (d) 1:7 TetC:(NeuAc) ${ }_{3}$.

\section{Role of Acetylneuraminic Acid in Carbohydrate Binding to Tetanus Toxin}

The $\mathrm{K}_{\mathrm{D}}$ values obtained for the NeuAc-containing carbohydrate-TetC complexes ranged between 11 to $33 \mu \mathrm{M}$ (Table 2). The dissociation constants obtained from the deconvoluted data were similar to those obtained from the unprocessed data. However, deconvolution of the $(\mathrm{NeuAc})_{6}$ and disialyllactose datasets was not possible due to poor signal to noise ratios, so only the results obtained from the unprocessed data is reported for these two cases.

The dissociation constants of TetC complexes with molecules containing a single NeuAc (NeuAc run at 200

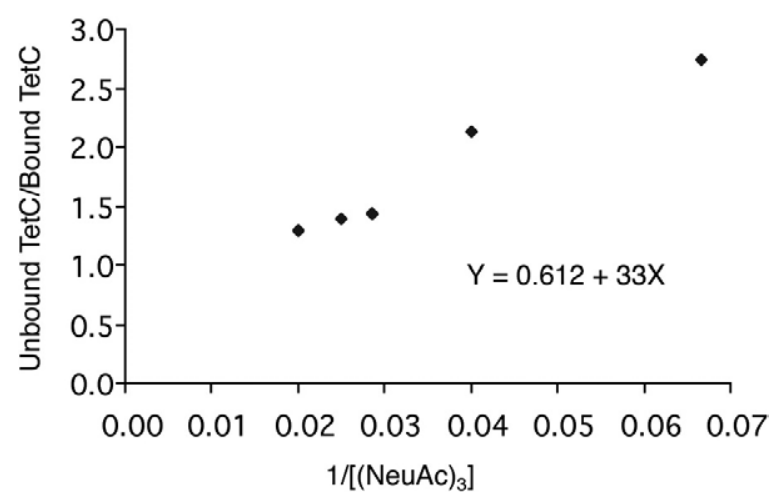

Figure 6. Binding of (NeuAc) $)_{3}$ to TetC. The dissociation constant $\mathrm{K}_{\mathrm{D}}$ is derived from the slope of the plot of the ratio of unbound TetC/bound TetC versus $1 /\left[(\mathrm{NeuAc})_{3}\right]$ (see eq 2 ).
$\mathrm{V}$, NeuAc run at $250 \mathrm{~V}$, and sialyllactose) were found to be very close in value $(11 \mu \mathrm{M}, 15 \mu \mathrm{M}$, and $13 \mu \mathrm{M}$, respectively). A very similar dissociation constant, 10 $\mu \mathrm{M}$, was reported previously for the binding of the $\mathrm{GM}_{3}$ ganglioside (one NeuAc) to TetC in an assay that monitored the inhibition of this protein binding to brain membranes [30]. The dissociation constants of TetC bound to molecules containing two NeuAc components (disialyllactose and $(\mathrm{NeuAc})_{2}$ ) were also found to be similar to each other ( $23 \mu \mathrm{M}$ and $21 \mu \mathrm{M}$, respectively). While the consistency in measured dissociation constants for carbohydrates containing one versus two NeuAc components obtained in this study suggests that binding to TetC is reduced upon addition of the second NeuAc, additional experiments need to be conducted using other techniques to confirm that the dissociation constants are different and that the presence of a second NeuAc residue destabilizes the interaction of the first residue within a particular binding site.

The largest dissociation constant was obtained for the TetC complex involving the NeuAc trimer (see below for binding of tetramer and hexamer), indicating that a longer oligomer does not interact as tightly with TetC. These differences in dissociation constants are subtle and could be misleading (it is generally accepted that two Kd values must differ by more than 2 -fold to be considered different). However, the general trend observed for the set of NeuAc oligomers appears consistent with both the molecular structure of the gangliosides and the location of sites on BoNT/B (Clostridium 
botulinum neurotoxin type B) and the $C$ fragment of tetanus toxin where NeuAc residues appear to bind. Carbohydrates in gangliosides that preferentially bind to the Clostridial neurotoxins (GT1b, GD1b) contain a single di NeuAc separated from another NeuAc (or two di NeuAc separated from each other as in $\mathrm{G}_{\mathrm{Q} 1 \mathrm{~b}}$ ) by Gal( $\beta 1-3)-G a l N A c(\beta 1-4)-G a l$ (Table 1). Crystallographic data obtained by Swaminathan and Eswaramoorthy [31] have shown that the NeuAc residue in sialyllactose binds in a cleft on the surface of the targeting domain of BoNT/B between Trp 1261 and His 1240, one of the potential ganglioside binding sites. A different X-ray crystallography study [32] showed that four separate carbohydrates, NeuAc, galactose, lactose, and N-acetylgalactosamine, bind to different sites of the $C$ fragment. Interestingly, a single NeuAc was found to bind in the region of residues 1226 to 1229 , adjacent to the position occupied by $\mathrm{N}$-acetylgalactosamine in a different crystal structure. Their results support the hypothesis that TetC contains multiple carbohydrate binding sites.

Recent competition experiments performed using NMR [34] have also revealed that sialic acid effectively competes with doxorubicin for binding to the cleft between Trp 1289 and His 1271. It has been shown [32] that even though the sequence homology between the $\mathrm{C}$ fragment of the Clostridial neurotoxins BoNT/A, BoNT/B, and tetanus (TeNT) is low, these domains seem to adopt the same type of folds. The observation that the C-fragment appears to have various sites capable of accommodating oligomeric carbohydrates [32] may explain the dissociation constants at the nanomolar level obtained when either tetanus or botulinum binds to neuronal cell membranes [30]. While it is not yet know whether or not the presence of multiple carbohydrate binding sites may lead to the binding of a single ganglioside to two neurotoxin proteins or the multivalent binding of the ganglioside to each protein, crosslinking appears to exist in the TeNT case [34]. No binding was observed with Gal-GalNAc in our MS experiments. However, Gal-GalNAc was shown to be required for binding to TeNT [34].

These results, combined with the information that the single NeuAc and the di NeuAc residues are separated by three galactose residues in GT1b (and GD1b, Table 1), suggest that ganglioside binding may involve at least two adjacent sites on the surface of the targeting domain of the Clostridial neurotoxins. If this is true, then one might expect the dissociations constants of a series of NeuAc oligomers to reflect the distance between these two binding sites. Our ESI-MS results appear to be consistent with this hypothesis. If we consider, for example, the binding of the first NeuAc of GT1b or GD1b into one of the two binding folds, the distance between this residue and the next NeuAc (or di NeuAc) binding in the second pocket would correspond to $\sim 25$ $\AA$ (based on the distance between the NeuAc residues in a ganglioside). This is very close to the distance that separates the two sites on the Clostridial neurotoxin targeting domains that have been identified to bind
NeuAc [34] and either sialyllactose [32] or an analog of the GT1b carbohydrate [35]. By analogy, if the NeuAc oligomers bound to these two sites, we would expect the strongest binding to be observed with the monomer and the hexamer. This is consistent with the binding data obtained in these experiments. While the monomer has a dissociation constant of $13 \mu \mathrm{M}$, the dimer, trimer, and pentamer appear to bind less tightly (larger dissociation constants). Once the length reached six residues, the $\mathrm{K}_{\mathrm{D}}$ appeared to decrease, suggesting that the hexamer may bind more tightly. Tetramer binding, which appears to be more complex (two slopes were observed with non-deconvolved spectra, on two separate titration experiments), does not follow the linear premise of this model. This may indicate that tetramer binding to the $C$ fragment may occur via an entirely different mode, one that reflects the ability of the tetramer to adopt a unique conformation not attainable by the other oligomers.

As the ratio of ligand to protein increased (1:5 for the monomer, dimer, and trimer, and 1:3 for the tetramer), a second ligand was observed to bind to TetC. These results provide additional support for the idea that the sialic acids may bind to two different sites on the surface of TetC. A complex containing two (NeuAc) ${ }_{6}$ or $(\mathrm{NeuAc})_{5}$ would be difficult to detect because the corresponding mass would not be adequately separated from the next charged state. Because of the complexities involved in having multiple TetC binding sites and the possibility of having more than one ligand bind to TetC, eqs 1 and 2, which are based on a simple one-ligand/ one-binding site model, would not apply at high ligand concentrations. This could explain why the linearity in Figure 6 has only a regression $\mathrm{R}^{2}$ of $0.95,841$.

Previous studies have clearly shown that TetC preferentially binds gangliosides of the series $\mathrm{G}_{\mathrm{T} 1 \mathrm{~b}}, \mathrm{G}_{\mathrm{Q} 1 \mathrm{~b}}$, $\mathrm{G}_{\mathrm{D} 1 \mathrm{~b}}$. The (NeuAc)$)_{2}$ group on the internal Gal of this ganglioside series (Table 1) has been reported to be required for binding, as well as the NeuAc on the terminal Gal( $\beta 1-3)$-GalNAc moiety [36, 37]. The single NeuAc in the internal Gal, as in $\mathrm{G}_{\mathrm{M} 1}$, did not appear to be sufficient for binding using a microtiter assay [36], but was observed to bind in competition experiments [37]. The NeuAc on the terminal Gal in $\mathrm{G}_{\mathrm{M} 3}$ appeared to enhance binding only slightly and was not considered essential for binding [36]. Our ESI-MS experiments, on the other hand, show that both sialyllactose (NeuAc $(\alpha 2-3) \mathrm{Gal}(\beta 1-4)$ Glc or $\mathrm{GM}_{3}$ carbohydrate) and disialyllactose (NeuAc $(\alpha 2-8)$ NeuAc $(\alpha 2-3)$ Gal $(\beta 1-4)$ Glc or $\mathrm{G}_{\mathrm{T} 1 \mathrm{~b}}$ fragment) bind to the TetC. The most likely explanation for the observed differences in binding is that the mass spectrometry technique (and competition assays) can be used to detect weakly bound ligands with micromolar dissociation constants. Such weak binding would not be detected using a microtiter assay because weaker binding ligands would dissociate and be removed in the washing steps.

The strength of the interaction between the tetanus toxin and gangliosides reported varies widely. Early 
studies reported the affinities between tetanus toxin and gangliosides to be in the low $\mu \mathrm{M}$ range [38]. Later studies reported that gangliosides compete for tetanus toxin binding to bovine cerebral cortex membranes with nanomolar potency [30]. A study using a quartz crystal microbalance measured binding constants of $\sim \mu \mathrm{M}$ between TetC and the gangliosides $\mathrm{G}_{\mathrm{D} 1 \mathrm{a}}, \mathrm{G}_{\mathrm{D} 1 \mathrm{~b}}$, and $\mathrm{G}_{\mathrm{T} 1 \mathrm{~b}}$ attached to solid supported membranes [38]. Another study using surface plasmon resonance and a liposome capture method obtained dissociation constants for $\mathrm{G}_{\mathrm{D} 1 \mathrm{~b}}$ and $\mathrm{G}_{\mathrm{T} 1 \mathrm{~b}}$ and the $\mathrm{C}$ fragment of 0.15 and $0.17 \mu \mathrm{M}$, respectively [39].

The dissociation constant measured for disialyllactose in the present study is about 100 times weaker than that obtained for $\mathrm{G}_{\mathrm{T} 1 \mathrm{~b}}$ by surface plasmon resonance [39]. This discrepancy may be due to difference in the concentrations used in each experiment [15], or more likely, structural differences in the carbohydrate components of the gangliosides or gangliosides tested. The additional NeuAc ( $\alpha 2-3)$ Gal( $\beta 1-3)$-GalNAc residue present in $\mathrm{G}_{\mathrm{T} 1 \mathrm{~b}}$, for example, may contribute to its stronger, more specific binding [30]. In addition, the membrane bilayer may play an important role in specific protein-carbohydrate interactions. The fluidity of the bilayer allows congregation of gangliosides and correct geometric positioning of their polar headgroups $[40,41]$. The monovalent interactions in toxin-ganglioside binding, which can be characterized by unusually weaker affinities, usually become strong interactions as a result of the multivalent interactions that occur through the simultaneous association of a single protein with multiple ganglioside molecules [42]. Additional stability and selectivity can be achieved when protein receptors specific to the cell participate in toxin binding.

The validity of using ESI-MS to measure dissociation constants between Clostridial neurotoxins and their receptors has recently been confirmed in another study [19]. Doxorubicin, a ligand that was found to bind to TetC, produced a dissociation constant of $11 \mu \mathrm{M}$ by the same electrospray technique used in this work. The complex could be dissociated in the gas phase by increasing the skimmer voltage, indicating that the association was possibly more hydrophobic in character than the interaction with the NeuAc ligands. A competitive assay using ganglioside-bearing liposomes produced a dissociation constant with a very similar value of $9.4 \mu \mathrm{M}$ for this complex [19].

\section{Conclusion}

ESI- MS methods have been successfully used to study the oligomeric state of TetC and the complex formation between TetC and a set of NeuAc containing ligands. Dissociation constants for the TetC/ligand complexes measured by ESI-MS are consistent with values obtained using other methods, demonstrating that ESI-MS can be used to study specific, noncovalent interactions between proteins and ligands. The dissociation constant obtained for disialyllactose was determined to be sub- stantially weaker than the binding constant measured previously for $G_{T 1 b}$, which suggests that the terminal $\operatorname{NeuAc}(\alpha 2-3) \operatorname{Gal}(\beta 1-3)-G a l N A c$ in $\mathrm{G}_{\mathrm{T} 1 \mathrm{~b}}$ (and possibly interactions with other protein toxin sites and/or among the carbohydrates) impart both greater specificity and higher affinity.

One of the disadvantages that was observed using ESI-MS is that the technique's ability to measure nM affinities might be compromised when ligand concentrations tested do not span the range well below and above the expected $\mathrm{K}_{\mathrm{D}}$, which in turn might be limited by instrument sensitivity. The strength of the interactions between $\mathrm{TetC}$ and (NeuAc) ${ }_{n}$ is consistent with the topography of the targeting domain of tetanus toxin and the distances between its carbohydrate binding sites. The results suggest that the targeting domain of tetanus toxin contains two binding sites that can accommodate NeuAc residues separated by a distance of $\sim 25$ $\AA$. This is consistent with the structure of $G_{T 1 b}$ and related gangliosides that bind to the tetanus toxin targeting domain where the NeuAc residues are separated by a similar distance.

\section{Acknowledgments}

The mass spectrometry work at UCSF was supported by NIH NCRR BRTP grant RR01614. The work conducted at Lawrence Livermore National Laboratory was supported by the Department of Energy's Chemical and Biological Non-Proliferation Program. RMW thanks the Natural Sciences and Engineering Research Council of Canada for the postdoctoral fellowship. MCPC thanks Professor Ronald Schnaar and his group (Johns Hopkins University) for stimulating discussions and helpful suggestions. This work was performed under the auspices of the U.S. Department of Energy by Lawrence Livermore National Laboratory under contract W-7405-ENG-48.

\section{References}

1. Niemann, H. Molecular Biology of Clostridial Neurotoxins. In Sourcebook of Bacterial Toxins 1991; Alouf, J. E.; Freer, J. E., Eds.; Academic: New York, pp 303-348.

2. Montecucco, C.; Schiavo, G. Structure and Function of Tetanus and Botulinum Neurotoxins. Quarterly Rev. Biophys. 1995, 28, 423-472.

3. Williamson, L. C.; Bateman, K. E.; Clifford, J. C. M.; Neale, E. A. Neuronal Sensitivity to Tetanus Toxin Requires Gangliosides. J. Biol. Chem. 1999, 274, 25173-25180.

4. Shapiro, R. E.; Specht, C. D.; Collins, B. E.; Woods, A. S.; Cotter, R. J.; Schnaar, R. L. Identification of a Ganglioside Recognition Domain of Tetanus Toxin using a Novel Ganglioside Photoaffinity Ligand. J. Biol. Chem. 1997, 272, 30380-30386.

5. Schengrund, C. L. What is the Cell Surface Receptor(s) for the Different Serotypes of Botulinum Neurotoxin? J. Toxicol. Toxin Rev. 1999, 18, 35-44.

6. Ganem, B.; Li, Y. T.; Henion, J. D. Detection of Noncovalent Receptor Ligand Complexes by Mass Spectrometry. J. Am. Chem. Soc. 1991, 113, 6294-6296.

7. Katta, V.; Chait, B. T. Observation of the Heme Globin Complex in Native Myoglobin by Electrospray-Ionization Mass-Spectrometry. J. Am. Chem. Soc. 1991, 113, 8534-8535.

8. Greig, M. J.; Gaus, H.; Cummins, L. L.; Sasmor, H.; Griffey, R. H. Measurement of Macromolecular Binding Using Electrospray Mass Spectrometry-Determination of Dissociation Constants for Oligonucleotide-Serum Albumin Complexes. J. Am. Chem. Soc. 1995, 117, 1076510766.

9. Loo, J. A. Studying Noncovalent Protein Complexes by Electrospray Ionization Mass Spectrometry. Mass Spectrom. Rev. 1997, 16, 1-23.

10. Smith, R. D.; Bruce, J. E.; Wu, Q. Y.; Lei, Q. P. New Mass Spectrometric Methods for the Study of Noncovalent Associations of Biopolymers. Chem. Soc. Rev. 1997, 26, 191-202. 
11. Veenstra, T. D. Electrospray Ionization Mass Spectrometry in the Study of Biomolecular Noncovalent Interactions. Biophys. Chem. 1999, 79, 63-79.

12. Chitta, R. K.; Rempel, D. L.; Gross, M. L. Determination of Affinity Constants and Response Factors of the Noncovalent Dimer of Gramicidin by Electrospray Ionization Mass Spectrometry. J. Am. Soc. Mass Spectrom. 2005, 16, 1031-1038.

13. Tjernberg, A.; Carno, S.; Oliv, F.; Benkestock, K.; Edlund, P. O.; Griffiths, W. J.; Hallen, D. Determination of Dissociation Constants for ProteinLigand Complexes by Electrospray Ionization Mass Spectrometry. Anal. Chem. 2004, 76, 4325-4331.

14. Robinson, C. V.; Chung, E. W.; Kragelund, B. B.; Knudsen, J.; Aplin, R. T.; Poulsen, F. M.; Dobson, C. M. Probing the Nature of Noncovalent Interactions by Mass Spectrometry. A Study of Protein-CoA Ligand Binding and Assembly. J. Am. Chem. Soc. 1996, 118, 8646-8653.

15. Sannes-Lowery, K. A.; Mei, H.-Y.; Loo, J. A. Studying Aminoglycoside Antibiotic Binding to HIV-1 TAR RNA by Electrospray Ionization Mass Spectrometry. Int. J. Mass Spectrom. 1999, 193, 115-122.

16. Peschke, M.; Verkerk, U. H.; Kebarle, P. Features of the ESI Mechanism that Affect the Observation of Multiply Charged Noncovalent Protein Complexes and the Determination of the Association Constant by the Titration Method. J. Am. Soc. Mass Spectrom. 2004, 15, 1424-1434.

17. Kebarle, P. A Brief Overview of the Present Status of the Mechanisms Involved in Electrospray Mass Spectrometry. J. Mass Spectrom. 2000, 35, $804-817$.

18. Wortmann, A.; Kistler-Momotova, A.; Willhelm, O.; Zenobi, R. Investigation of Shifts in Chemical Equilibria in Shrinking Droplets of an Electrospray Plume. Poster ThP 231. Proceedings of the 53rd ASMS Conference; San Antonio, TX, June, 2005.

19. Lightstone, F. C.; Prieto, M. C.; Singh, A. K.; Piqueras, M. C.; Whittal, R. M.; Knapp, M. S.; Balhorn, R.; Roe, D. C. Identification of Novel Small Molecule Ligands that Bind to Tetanus Toxin. Chem. Res. Toxicol. 2000, 13, 356-362.

20. Whittal, R. M.; Ball, H. L.; Cohen, F. E.; Burlingame, A. L.; Prusiner, S. B.; Baldwin, M. A. Copper Binding to Octarepeat Peptides of the Prion Protein Monitored by Mass Spectrometry. Protein Sci. 2000, 9, 332-343.

21. Whittal, R. M.; Benz, C. C.; Scott, G.; Semyonov, J.; Burlingame, A. L.; Baldwin, M. A. Preferential Oxidation of Zinc Finger 2 in Estrogen Receptor DNA-Binding Domain Prevents Dimerization and, hence, DNA Binding. Biochemistry 2000, 39, 8406-8417.

22. Hoch, D. H.; Romeromira, M.; Ehrlich, B. E.; Finkelstein, A.; Dasgupta, B. R.; Simpson, L. L. Channels Formed by Botulinum, Tetanus, and Diphtheria Toxins in Planar Lipid Bilayers-Relevance to Translocation of Proteins across Membranes. Proc. Nat. Acad. Sci. U.S.A. 1985, 82, of Proteins

23. Gambale, F.; Montal, M. Characterization of the Channel Properties of Tetanus Toxin in Planar Lipid Bilayers. Biophys. I. 1988, 53, 771-783.

24. Montal, M. S.; Blewitt, R.; Tomich, J. M.; Montal, M. Identification of an Ion Channel-Forming Motif in the Primary Structure of Tetanus and Botulinum Neurotoxins. FEBS Lett. 1992, 313, 12-18.

25. Schmid, M. F.; Robinson, J. P.; Dasgupta, B. R. Direct Visualization of Botulinum Neurotoxin-Induced Channels in Phospholipid-Vesicles. $\mathrm{Na}$ ture 1993, 364, 827-830.

26. Ledoux, D. N.; Be, X. H.; Singh, B. R. Quaternary Structure of Botulinum and Tetanus Neurotoxins as Probed by Chemical Cross-Linking and Native Gel-Electrophoresis. Toxicon 1994, 32, 1095-1104.
27. Jorgensen, T. J. D.; Roepstorff, P.; Heck, A. J. R. Direct Determination of Solution Binding Constants for Noncovalent Complexes Between Bacterial Cell Wall Peptide Analogues and Vancomycin Group Antibiotics by Electrospray Ionization Mass Spectrometry. Anal. Chem. 1998, 70, 4427-4432.

28. Lim, H. K.; Hsieh, Y. L.; Ganem, B.; Henion, J. Recognition of Cell-Wall Peptide Ligands by Vancomycin Group Antibiotics-Studies Using Ion-Spray Mass Spectrometry. I. Mass Spectrom. 1995, 30, 708-714.

29. Vandorsselaer, A.; Bitsch, F.; Green, B.; Jarvis, S.; Lepage, P.; Bischoff, R.; Kolbe, H. V. J.; Roitsch, C. Application of Electrospray Mass-Spectrometry to the Characterization of Recombinant Proteins up to $44 \mathrm{Kda}$. Biomed. Environ. Mass Spectrom. 1990, 19, 692-704.

30. Rogers, T. B.; Snyder, S. H. High-Affinity Binding of Tetanus Toxin to Mammalian Brain Membranes. J. Biol. Chem. 1981, 256, 2402-2407.

31. Swaminathan, S.; Eswaramoorthy, S. Structural Analysis of the Catalytic and Binding Sites of Clostridium botulinum Neurotoxin B. Nat. Struct. Biol. 2000, 7, 693-699.

32. Emsley, P.; Fotinou, C.; Black, I.; Fairweather, N. F.; Charles, I. G.; Watts, C.; Hewitt, E.; Isaacs, N. W. The Structures of the H-C Fragment of Tetanus Toxin with Carbohydrate Subunit Complexes Provide Insight into Ganglioside Binding. J. Biol. Chem. 2000, 275, 8889-8894.

33. Cosman, M.; Lightstone, F. C.; Krishnan, V. V.; Zeller, L.; Prieto, M. C. Roe, D. C.; Balhorn, R. Identification of Novel Small Molecules that Bind to Two Different Sites on the Surface of Tetanus Toxin C Fragment. Chem. Res. Toxicol. 2002, 15, 1218-1228.

34. Fotinou, C.; Emsley, P.; Black, I.; Ando, H.; Ishida, H.; Kiso; Sinha, K. A.; Fairweather, N. F.; Isaacs, N. W. The Crystal Structure of Tetanus Toxin HC Fragment Complexed with a Synthetic GT1b Analog Suggests Crosslinking Between Ganglioside Receptors and the Toxin. J. Biol. Chem. 2001, 276, 32274-32281.

35. Angstrom, J : Teneberg, S.; Karlsson, K. A. Delineation and Comparison of Ganglioside-Binding Epitopes for the Toxins of Vibrio cholerae, Escherichia coli, and Clostridium tetani-Evidence for Overlapping Epitopes. Proc. Natl. Acad. Sci. U.S.A. 1994, 91, 11859-11863.

36. Holmgren, J. Elwing, H.; Fredman, P.; Svennerholm, L. PolystyreneAdsorbed Gangliosides for Investigation of the Structure of the Tetanus-Toxin Receptor. Eur. J. Biochem. 1980, 106, 371-379.

37. Ledley, F. D.; Lee, G.; Kohn, L. D.; Habig, W. H.; Hardegree, M. C. Tetanus Toxin Interactions with Thyroid Plasma-Membranes-Implications for Structure and Function of Tetanus Toxin Receptors and Potential Pathophysiological Significance. J. Biol. Chem. 1977, 252, 4049 4055 .

38. Janshoff, A.; Steinem, C.; Sieber, M.; elBaya, A.; Schmidt, M. A.; Galla, H. J. Quartz Crystal Microbalance Investigation of the Interaction of Bacterial Toxins with Ganglioside Containing Solid Supported Membranes. Eur. Biophys. J. Biophys. Lett. 1997, 26, 261-270.

39. MacKenzie, C. R.; Hirama, T.; Lee, K. K.; Altman, E.; Young, N. M. Quantitative Analysis of Bacterial Toxin Affinity and Specificity for Glycolipid Receptors by Surface Plasmon Resonance. J. Biol. Chem. 1997, 272, 5533-5538.

40. Evans, S. V.; MacKenzie, C. R. Characterization of Protein-Glycolipid Recognition at the Membrane Bilayer J. Mol. Recognit. 1999, 12, 155-168.

41. Brocca, P.; Sonnino, S. Dynamics and Spatial Organization of Surface Gangliosides. Trends Glycosci. Glycotechnol. 1997, 9, 433-445.

42. Winter, A.; Ulrich, W. P.; Wetterich, F.; Weller, U.; Galla, H. J. Gangliosides in Phospholipid Bilayer Membranes: Interaction with Tetanus Toxin. Chem. Phys. Lipids 1996, 81, 21-34. 